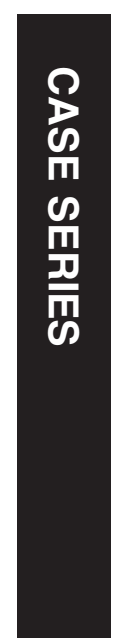

${ }^{1}$ Oxford Eye Hospital, John Radcliffe Hospital, Oxford, UK

${ }^{2}$ Department of Rheumatology, Nuffield Orthopaedic Centre, Oxford, UK

Correspondence: SM Downes, Oxford Eye Hospital, John Radcliffe Hospital, Oxford OX3 9DU, UK

Tel: 01865 234735;

Fax: 01865234515.

E-mail: susan.downes@eye. ox.ac.uk

Received: 4 July 2016 Accepted in revised form: 23 November 2016

Published online:

10 February 2017

\section{Hydroxychloroquine retinopathy: an emerging problem}

M Latasiewicz ${ }^{1}$, H Gourier ${ }^{1}$ IH Yusuf ${ }^{1}$, R Luqmani ${ }^{2}$, SM Sharma ${ }^{1}$ and SM Downes ${ }^{1}$ improved safety profile compared with its predecessor, chloroquine, it is now becoming clearer that retinal toxicity is a side effect of prolonged use and is emerging as a significant cause of visual loss for susceptible patients on this therapy.

Retinopathy caused by antimalarials, first described in $1963,{ }^{1}$ is usually asymptomatic, and by the time symptoms of photosensitivity and patchy visual loss develop, retinal changes are already present. The introduction of more sophisticated imaging has enabled the identification of pre-symptomatic retinal changes indicative of toxicity. ${ }^{2,3}$

\section{Case 1}

A 63-year-old caucasian female presented with glare and decreased vision for 9 months having taken HCQ $400 \mathrm{mg}$ per day for RA for 16 years (cumulative dose of $2336 \mathrm{~g} ; 4.93 \mathrm{mg} / \mathrm{kg}$ per day). Her medications included omeprazole, methotrexate, and folic acid. Her renal function was $84 \mathrm{ml} / \mathrm{min} / 1.73 \mathrm{~m}^{2}$. Visual acuities were 6/6 right and 6/7.4 left. Fundoscopy and imaging revealed subtle disturbances of the macular retinal pigment epithelium, increased AF signal on Scanning Laser Ophthalmoscopy (SLO), with bilateral parafoveal thinning on Spectral Domain OCT imaging (Figures 1a and $b$, Figures $2 a$ and $b) .{ }^{4}$ Ring scotomata were identified on visual field testing (Figures $3 a$ and b); electrophysiology demonstrated normal rod and cone responses, but abnormal macular function. Repeat testing 6 months later revealed stable visual acuities and no deterioration on electrophysiological testing.

\section{Case 2}

A 44-year-old caucasian female noted decreased vision and central scotomas 6 months prior to presentation, having taken a daily dose of HCQ $400 \mathrm{mg}$ for RA for 15 years (cumulative dose $2910 \mathrm{~g} ; 5.6 \mathrm{mg} / \mathrm{kg}$ per day). Her medications included leflunomide, methotrexate, 
omeprazole, loratadine, folic acid. Her renal function was $84 \mathrm{ml} / \mathrm{min} / 1.73 \mathrm{~m}^{2}$. Visual acuities were $6 / 6$ in both eyes. Fundoscopy and imaging revealed bilateral Bull's eye maculopathy with typical AF and OCT findings
(Figures 1c and d, Figures 2c and d). Visual field testing showed central sensitivity losses with decreased macular function on the multifocal ERG. (Figures $3 \mathrm{c}$ and $\mathrm{d}$ ).

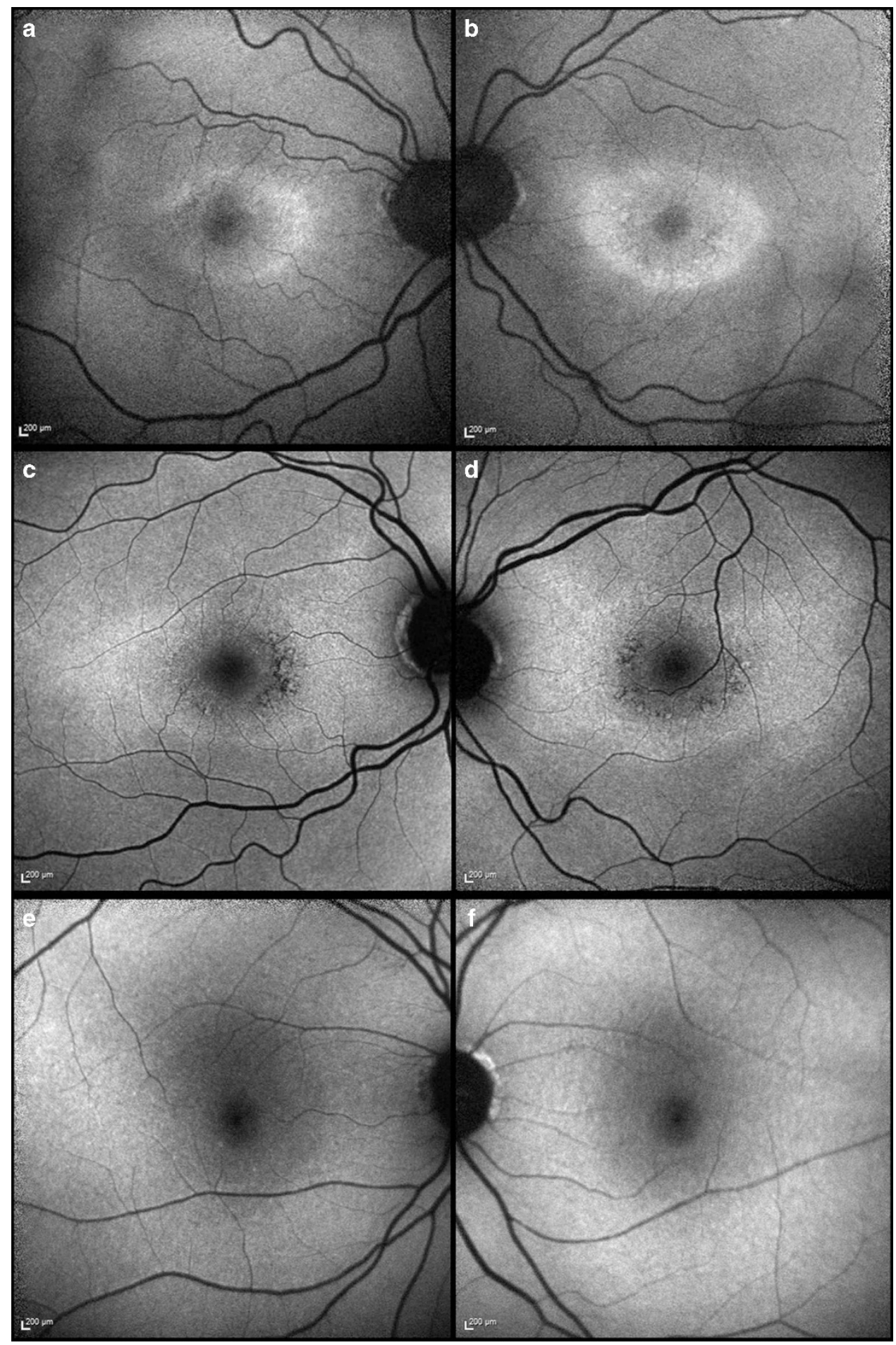

Figure 1 Autofluorescence (AF) imaging in HCQ retinopathy. (a, b) Case 1 demonstrates a significant bilateral increase in parafoveal AF signal. (c, d) Case 2 demonstrates a more widespread increase in AF signal with patchy decreasing signal parafoveally indicative of atrophic change bilaterally. (e, f) Case 3 demonstrates very minimal changes on AF. 


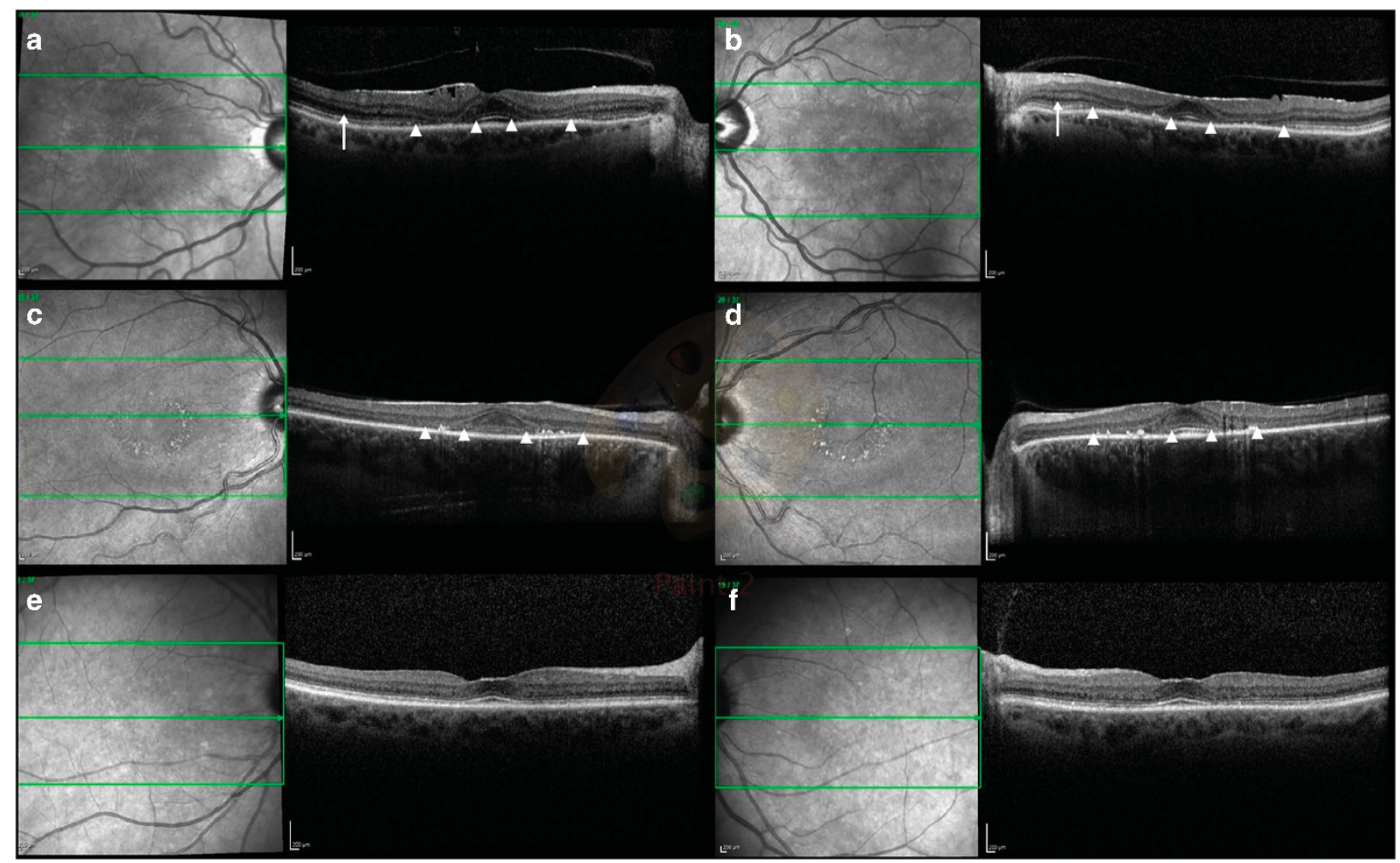

Figure 2 The OCT features of HCQ retinopathy in severe and mild retinopathy. (a, b) Case 1 demonstrates parafoveal thinning of outer retinal segments in both eyes with disruption of the ellipsoid zone and photoreceptor inner and outer segments (between white arrowheads). Epiretinal membrane formation is noted, more pronounced in the right eye. Posterior vitreous separation is seen bilaterally. The external limiting membrane is not visualised in the parafovea but is seen more peripherally (arrow). (c, d) Case 2 demonstrates bilateral parafoveal thinning of the ellipsoid zone and photoreceptor inner and outer segments (between white arrowheads) with corresponding retinal pigment epithelium changes on scanning laser ophthalmoscopy. The external limiting membrane is not visualised. (e, f) Case 3 demonstrates fine retinal pigment epithelium changes. The external limiting membrane is preserved.

\section{Case 3}

A 74-year-old caucasian female with SLE presented with a 4 month history of bilateral central scotomata in the last 4 months, having taken $400 \mathrm{mg}$ of HCQ per day for 16 years, followed by $200 \mathrm{mg}$ per day for 6 months (total dose of $2372 \mathrm{~g} ; 4.93 \mathrm{mg} / \mathrm{kg}$ per day (on $400 \mathrm{mg}$ per d)). Her medications included losartan, atorvastatin, furosemide, fenofibrate, colchicine, and probenecid. She had not previously taken tamoxifen. She had chronic kidney disease (stage 3) with a 4-year history of deteriorating renal function: her eGFR at the time of diagnosis was $28 \mathrm{ml} / \mathrm{min} / 1.73 \mathrm{~m}^{2}$. Visual acuities were $6 / 6$ in both eyes. Imaging and functional testing confirmed a central maculopathy (Figures 1e and f, Figures 2e and f, Figures $3 e$ and f).

\section{Discussion}

HCQ is a widely used drug found to be of significant benefit in rheumatological and dermatological conditions, with demonstrated survival benefits for SLE. ${ }^{5}$ However, like Chloroquine, where a similar bull's eye maculopathy develops, HCQ has now also been shown to be associated with significant retinal toxicity in susceptible individuals. While older studies have estimated HCQ toxicity to be infrequent at $\sim 0.5-1 \%$ of those taking HCQ for greater than 5 years, ${ }^{6}$ recent epidemiological data indicate that retinal toxicity occurs in greater than $10 \%$ of patients, who have taken HCQ for over 10 years, and $20-50 \%$ of patients taking HCQ for greater than 20 years. ${ }^{7}$

The 2009 Royal College of Ophthalmologists Guidelines on HCQ screening advise referral to an Ophthalmologist only if the patient has baseline visual impairment, eye disease confirmed by an optometrist, or if the patient notices visual symptoms. ${ }^{8}$ This was based on the premise that the introduction of HCQ was a safe option and was not thought to be associated with the same toxicity as chloroquine. Patients were previously instructed to selfmonitor with an Amsler grid, but regular screening has not been common practice in the UK. 
a
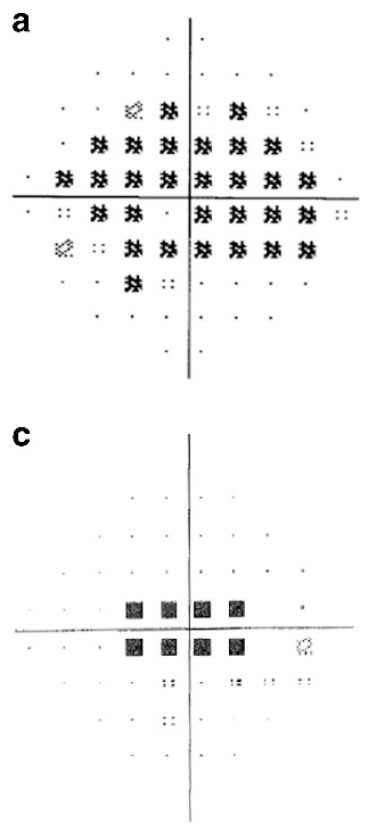

e

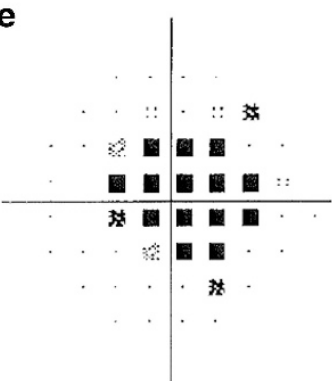

b

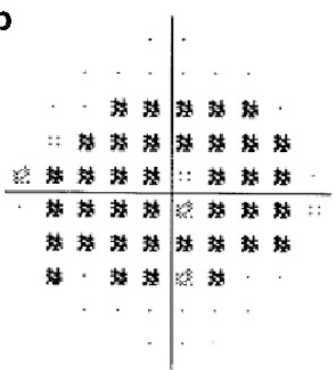

d

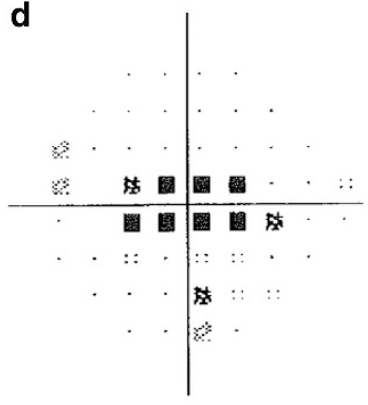

$\mathbf{f}$

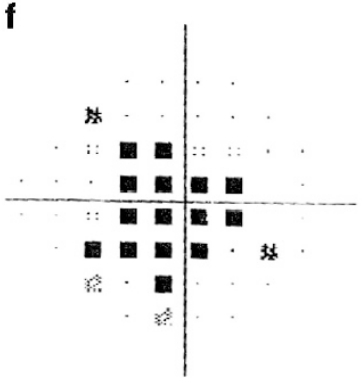

Figure 3 Visual field exams in HCQ retinopathy. All presented plots are those from Humphrey automated perimetry of the central field (10-2). (a, b) Case 1 demonstrates characteristic ring scotomas. (c, d) Case 2 demonstrates central scotomas. (e, f) Case 3 demonstrates central visual field defects.

The American Academy of Ophthalmology (AAO) 2016 guidelines recommend baseline ophthalmologic examination including fundoscopy with further testing if abnormalities are present at baseline, as well as annual automated central visual field testing (Humphrey 10-2), SD-OCT, and AF after 5 years of exposure or sooner. ${ }^{3}$ Amsler grid monitoring was not included in the recommendations because it was regarded as subjective and unreliable.

It is now clear that visual acuities, 10-2 visual fields, and imaging with OCT and FAF and if indicated, multifocal ERG, should be used to look for presymptomatic retinal toxicity. 3,7 Some patients' retinopathy progresses despite stopping treatment; involvement of the external limiting membrane on OCT carries negative prognostic value, suggestive of irreversible photoreceptor damage. ${ }^{9}$ We review the

specific retinal imaging and functional testing features in more detail in the accompanying review. ${ }^{10}$ Asian patients demonstrate a pericentral pattern of disease, and wider field imaging and visual field testing (30-2) may be required in these patients. ${ }^{11,12}$

Rheumatologists, Ophthalmologists and General practitioners who are involved in the care of these patients need to be aware of the need for initial screening and regular monitoring of patients. These cases highlight the importance of recognising and managing this emerging problem. Instituting a standardised screening protocol would enable cessation of therapy as early as possible, thus reducing the risk and extent of retinal toxicity. In the accompanying review, ${ }^{10}$ we present a proposal for screening and monitoring, which includes practical suggestions for imaging and functional testing.

\section{Summary}

What was known before

- Long-term treatment with chloroquine had been reported to cause severe irreversible retinal toxicity in susceptible individuals, which can progress despite discontinuation of the medication. Hitherto this had only been rarely reported in HCQ.

What this study adds

- This case series highlights the need to raise awareness of the potential retinal toxicity of HCQ.

- Specific features of HCQ toxicity on OCT, AF and visual field testing are described.

- The need for a standardised screening protocol to monitor for retinal toxicity in patients undergoing HCQ treatment is highlighted.

- Screening modalities are proposed.

\section{Conflict of interest}

The authors declare no conflict of interest.

\section{References}

1 Grupper C, Bregeat P, Juge P. [SEvere retinopathy during treatment of lupus erythematosus by synthetic antimalarials (2 cases)]. Bull Soc Fr Dermatol Syphiligr 1963; 70: 824-832.

2 Nika M, Blachley TS, Edwards P, Lee PP, Stein JD. Regular examinations for toxic maculopathy in long-term chloroquine or hydroxychloroquine users. JAMA Ophthalmol 2014; 132: 1199-1208.

3 Marmor MF, Kellner U, Lai TY, Melles RB, Mieler WF. Recommendations on screening for chloroquine and hydroxychloroquine retinopathy (2016 revision). Ophthalmology 2016; 123: 1386-1394.

4 Chen E, Brown DM, Benz MS, Fish RH, Wong TP, Kim RY et al. Spectral domain optical coherence tomography as an effective screening test for hydroxychloroquine retinopathy (the 'flying saucer' sign). Clin Ophthalmol 2010; 4: 1151-1158. 
5 Alarcon GS, McGwin G, Bertoli AM, Fessler BJ, Calvo-Alen J, Bastian HM et al. Effect of hydroxychloroquine on the survival of patients with systemic lupus erythematosus: data from LUMINA, a multiethnic US cohort (LUMINA L). Ann Rheum Dis 2007; 66: 1168-1172.

6 Mavrikakis I, Sfikakis PP, Mavrikakis E, Rougas K, Nikolaou A, Kostopoulos C et al. The incidence of irreversible retinal toxicity in patients treated with hydroxychloroquine: a reappraisal. Ophthalmology 2003; 110: 1321-1326.

7 Melles RB, Marmor MF. The risk of toxic retinopathy in patients on long-term hydroxychloroquine therapy. JAMA Ophthalmol 2014; 132: 1453-1460.

8 The Royal College of Ophthalmologists. Hydroxychloroquine and Ocular Toxicity Recommendations on Screening, The Royal College of Ophthalmologists, 2009. Available at: https:// www.rcophth.ac.uk/wp-content/uploads/2014/12/2009SCI-010-Ocular-Toxicity.pdf (accessed on 16 September 2016).

9 Mititelu M, Wong BJ, Brenner M, Bryar PJ, Jampol LM, Fawzi AA. Progression of hydroxychloroquine toxic effects after drug therapy cessation: new evidence from multimodal imaging. JAMA Ophthalmol 2013; 131: 1187-1197.

10 Yusuf IH, Sharma S, Luqmani R, Downes SM. Hydroxychloroquine retinopathy. Eye 2017 (in press); doi:10.1038/eye.2016.298.

11 Melles RB, Marmor MF. Pericentral retinopathy and racial differences in hydroxychloroquine toxicity. Ophthalmology 2015; 122: 110-116.

12 Lee DH, Melles RB, Joe SG, Lee JY, Kim JG, Lee CK et al. Pericentral hydroxychloroquine retinopathy in Korean patients. Ophthalmology 2015; 122: 1252-1256. 